\title{
Characteristics of women screened for a contraceptive intravaginal ring study in Kisumu, Kenya, 2014
}

\begin{abstract}
Eleanor McLellan-Lemal ${ }^{1+*}$, Deborah A. Gust ${ }^{1 \dagger}$, Roman Gvetadze $^{1 \dagger}$, Melissa Furtado ${ }^{1,2 \dagger}$, Fredrick O. Otieno ${ }^{3 \dagger}$, Mitesh Desai $^{1}$, Clement Zeh $^{4}$, Taraz Samandari' ${ }^{1}$, Beatrice Nyagol ${ }^{5}$ and Esther M. Makanga ${ }^{5}$
\end{abstract}

*Correspondence: egm4@cdc.gov

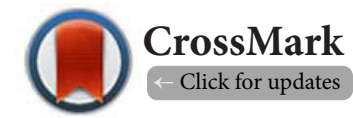

'These authors contributed equally to this work. ${ }^{1}$ Centers for Disease Control and Prevention, Office of Infectious Diseases, National Center for HIV/AIDS, Viral Hepatitis, STD, and TB Prevention, Division of HIV/AIDS Prevention, Atlanta, Georgia.

${ }^{2}$ Engility Corporation, Atlanta, GA, USA.

${ }^{3}$ Nyanza Reproductive Health Society, Kisumu, Kenya.

${ }^{4}$ Centers for Disease Control and Prevention, Office of Infectious Diseases, National Center for HIV/AIDS, Viral Hepatitis, STD, and TB Prevention, Division of HIV/AIDS Prevention, Kisumu, Kenya.

${ }^{5}$ Kenya Medical Research Institute, Kisumu, Kenya.

\begin{abstract}
Background: HIV antiretroviral-based intravaginal rings with and without co-formulated contraception hold promise for increasing HIV prevention options for women. Acceptance of and ability to correctly and consistently use this technology may create challenges for future ring-based microbicide trials in settings where this technology has not been introduced. We examined baseline factors associated with enrolling in a contraceptive intravaginal ring study in Kisumu, Kenya and describe notional acceptability (willingness to switch to a contraceptive ring based solely on information received about it).
\end{abstract}

Methods: Demographic, psychosocial, and behavioral eligibility screening of women 18-34 years was undertaken. Testing for pregnancy, HIV, and other sexually transmitted infections (STIs) was also conducted. We compared enrollment status across groups of categorical predictors using prevalence ratios (PR) and 95\% confidence interval (CI) estimates obtained from a log-binomial regression model.

Results: Out of 692 women pre-screened April to November 2014, 463 completed screening, and 302 women were enrolled. Approximately $97 \%$ of pre-screened women were willing to switch from their current contraceptive method to use the intravaginal ring exclusively for the 6-month intervention period. Pregnancy, HIV, and STI prevalence were $1.7 \%, 14.5 \%$, and $70.4 \%$ respectively for the 463 women screened. Women $18-$ 24 ( $\mathrm{PR}=1.47$, CI 1.15-1.88) were more likely to be enrolled than those 30-34 years of age, as were married/ cohabitating women (PR=1.62, CI 1.22-2.16) compared to those separated, divorced, or widowed. In adjusted analyses, sexual debut at less than 17 years of age, one lifetime sexual partner, abnormal vaginal bleeding in the past 12 months, condomless vaginal or anal sex in the past 3 months, and not having a sexual partner of unknown HIV status in the past 3 months were predictive of enrollment.

Conclusion: High notional acceptability suggests feasibility for contraceptive intravaginal ring use. Factors associated with ring use initiation and 6-month use will need to be assessed.

Keywords: Women, reproductive health, contraceptive intravaginal ring, Kenya, biomedical technology, sexual behavior, pregnancy, HIV and STI prevalence

\section{Introduction}

An integrated sexual and reproductive health approach, with emphasis on multipurpose prevention technologies (MPTs), is believed to offer the best solution for addressing women's needs [1]. Among the multiple MPTs under development, intravaginal rings (IVRs) have tremendous potential for preventing pregnancy, HIV, and other sexually transmitted infections (STIs) [2]. Only two IVRs are licensed for contraceptive use, neither 
of which are available currently in Kenya. Acceptance of andability to correctly and consistently use IVRs in this setting are largely unknown.

Vaginal delivery of hormonal contraceptives and antimicrobials avoids the need for daily administration, circumvents systemic absorption, limits required doses by avoiding hepatic first-pass metabolism, and can be used by women discreetly [3]. Correct and consistent IVR use, however, may be threatened by complex cultural, behavioral, physiological, physical, interpersonal, and structural issues that may not be recognized or are acknowledged but downplayed during clinical development [4-6]. Such factors include, but are not limited to, sexual practices, intravaginal hygiene and menstruation practices, side effect concerns or experiences, willingness to disclose use to others, reproductive intentions, partner support, vaginal comfort, interference during intercourse, hormonal side effects (nausea, headaches, gastrointestinal symptoms, vaginal discharge), the ring getting lost in the body [7-10], and IVR properties (e.g., method of insertion, duration of use, color, smell, size) [11].

Studies of NuvaRing, a one-month, low-dose etonogestrel and ethinyl estradiol-based ring, and other IVRs in development have suggested high product acceptability $[\mathbf{7}, \mathbf{8}, 10,12-14]$ with user satisfaction centered on a woman being able to control ring insertion and removal, absence of remembering to take a daily pill, and comfort and ease of use [15].

A fundamental question in introducing MPT IVR is whether women in developing countries are interested in such a product and its intended use(s). In this paper, we examined factors associated with enrolling in a study of NuvaRing use and describe notional acceptability of a contraceptive IVR (i.e., willingness to use a product solely based on information received about it).

\section{Material and methods Design}

Between April and November 2014, we enrolled women in a single group observational study of NuvaRing. Our research design included a pre-product phase ranging from 1 to 3 months (based on oral or injectable contraceptive use at enrollment) that was followed by 6 months of NuvaRing use, a one month post-product phase during which women returned to oral, injectable, or another contraceptive method of their choice, and then exited the study. For this analysis, we focused exclusively on screening data.

A multidisciplinary team of recruiters, data collectors, HIV test counselors, and study clinicians culturally similar to the target population and fluent in the three languages primarily spoken in the area (i.e., English, Kiswahili, and Dholuo) oversaw implementation of the study. The study staff was predominately comprised of women. All pelvic examinations were performed by female clinicians.

\section{Ethical review}

Review and approval of the study protocol, consent forms, and data collection instruments was completed by the Scientific Steering and Ethical Review Committees of the Kenya Medical Research Institute, and an Institutional Review Board for the United States (US) Centers for Disease Control and Prevention. This trial is registeredwith ClinicalTrials.gov numberNCT02529683.

Written informed consent was completed by women in their language preference before participating in data and specimen collections. Women who completed the in-depth screening process received a bar of soap, 500 Kenya Shillings (approximately \$5 US dollars) for transport, feminine sanitary pads, and a treated malaria bed net. No incentives were provided for the pre-screening eligibility assessment conducted in the recruitment venues.

Using convenience sampling, women were recruited from family planning and reproductive health clinics, via 10 community health workers, and participant word-of-mouth referrals without incentives. Based on initial community feedback, an overview of the study was presented to women in groups as opposed to approaching women individually. Women received information on the study, its purpose, and the risks and benefits of an IVR. They were shown a sample of the ring, allowed to visually and manually inspect it, and a 3-dimensional female reproductive model was used to demonstrate ring insertion and removal.

\section{Eligibility and data collection}

A two-step screening process (pre-screening and screening) was used. After privately obtaining pre-screening written informed consent and being assigned a unique study identification number, recruitment staff administered a brief pre-screening computer-assisted personal interview (CAPI). A woman was eligible to proceed with screening if she was 18 to 34 years of age, lived within 150 kilometers of Kisumu City, was sexually active in the past three months on more than one occasion, had used injectable depot medroxy-progesterone acetate (DMPA) or oral contraceptive pills (OCPs) in the past three months, and had never received an HIV-positive test result. Women also had to report willingness to switch from their existing birth control method to using NuvaRing for six months, to undergo periodic pelvic examinations and testing for pregnancy, HIV and other STIs, and to provide family clinic documentation of DMAP or OCP use in the past 3 months, as well as standard national documentation of age (e.g., identify card, birth notification/certificate).

Eligible women scheduled for a clinic screening visit, in which they presented the aforementioned documents and completed a second, more comprehensive written-informed consent that covered study risks, benefits, participant requirements, and procedures specifically related to screening and the pre-product phase. Detailed contact information was gathered and demographic, psychosocial, and behavioral information collected using audio computer-assisted selfinterview (ACASI). A study clinician administered a medical 
evaluation CAPI and performed a general physical examination as well as a pelvic examination. A female 3 -dimensional reproductive model was used to describe and demonstrate what would happen during the pelvic examination, and concerns were addressed before initiating the examination. Venous blood, urine, saliva and cervicovaginal lavage specimen collection was undertaken to test for pregnancy, HIV, herpes simplex virus type 2 (HSV-2), gonorrhea, syphilis, chlamydia, and bacterial vaginosis (BV). Verification that there were no pre-existing reproductive tract conditions was done through hematological and biochemistry analysis (e.g., cervical cancer visual inspection screening was completed using acetic acid and Lugol's iodine). Rapid HIV testing was performed with pre- and post- test counselling and results provided according to Kenyan Ministry of Health guidelines [16]. Women were encouraged but not required to disclose potential study participation to sexual partners.

A follow-up appointment was made within two weeks of the screening visit to permit clinical staff to review laboratory results and make a final study eligibility determination. Women were not eligible to participate if they were found to have current or a history of known medical contraindications for NuvaRing use (e.g., thrombophlebitis or thromboembolic disorders, cerebral vascular or coronary artery disease, valvular heart disease with thrombogenic complications, severe hypertension, diabetes with vascular involvement, headaches with focal neurological symptoms), to be breastfeeding or within three months of parturition, or tested positive for HIV. Women who tested HIV positive were provided additional counseling, underwent CD4 and viral load testing, and referred to a patient support center for appropriate HIV care and treatment services. Women who tested positive for gonorrhea, syphilis, or chlamydia were provided treatment and encouraged to invite their sexual partners to come for STI management and treatment [16]. Eligible women who declined study participation were asked to complete a refusal CAPI questionnaire.

\section{Measures}

Enrollment status ( $1=$ enrolled, $0=$ not enrolled) was our outcome. ACASI demographic variables included age group, ethnic/tribal group, marital status, religion, highest level of education completed, employment status, main source of income, and number of children in the household.

Notional acceptability, with dichotomous scores ( $1=y e s$, $0=$ no), was based on the CAPI pre-screening question, Are you willing to change from <current contraceptive method $>$ to using a vaginal ring to avoid or delay pregnancy? Notional acceptability was viewed as pre-product use acceptance given that actual product use would be undertaken 1-3 months post enrollment as opposed to hypothetical willingness, in which intentionality may not be specific to a particular product (brand or formulation) or future timeframe. Notional acceptability was operationalized as willingness to use NuvaRing after receiving detailing information about it, being given the opportunity to visually and manually inspect the ring, and being shown how it was inserted and removed using a 3-dimensional female reproductive model.

Psychosocial variables, with dichotomous scores ( $1=y e s$, $0=$ no), were based on questions on motivations for participation, pregnancy intentions/desires, contraception use barriers, and willingness to undergo periodic testing for pregnancy, HIV, and other STIs. Pelvic exam acceptance items adopted from Fiddes and colleagues [17] were rated on a 5-point Likert scale ( $1=$ strongly agree, $2=$ agree, $3=$ undecided, $4=$ disagree, and $5=$ strongly disagree) and a participant-level mean score was generated. The response scale for four negatively worded items (find pelvic exam unpleasant but can tolerate, anxious about the pelvic exam, distressed about the pelvic exam, would refuse the pelvic exam if offered) was reversed before scoring. Higher mean scores indicated less acceptance of/greater concern about pelvic exams. Cut-points for pelvic exam acceptance categories were derived from the quartiles for the pelvic exam measure ( $m e a n=2.8$ and median $=3.0 ;$ minimum $=0.7$ and maximum $=4.5$; lower quartile $=2.7$ and upper quartile=3.0). Three acceptance cut-points (mean score $\leq 2.7=$ high acceptance, mean score 2.7-2.99=medium acceptance, and mean score $\geq 3=$ low acceptance) were established.

Behavioral variables were age at sexual debut, number of sex partners (lifetime and in the past 3 months), history of forced sex, HIV-positive partner in the past 3 months, partner of unknown HIV status in the past 3 months, exchange sex in the past 3 months, vaginal or anal sex in without a condom in the past 3 months, history of having sex during menses, past history of STI diagnosis, alcohol use in the past 30 days, ever used drugs for recreational purposes, abnormal vaginal bleeding in the past 12 months, and past medication-taking history. Laboratory results for pregnancy, HIV, and STIs were also included.

\section{Statistical analysis}

We computed frequency counts and percentages to describe the demographics, psychosocial, and behavioral characteristics of women screened. In a univariable analysis, we compared enrollment status across groups of categorical predictors using prevalence ratios obtained from a log-binomial regression model. Adjusted effect estimates with $95 \%$ robust confidence intervals were obtained in a multivariable Poisson regression using the generalized estimating equations (GEE) approach. We employed backward elimination procedure with a 0.2 threshold level to select covariates in multivariable regression. All analyses were performed in SAS 9.3 (SAS Institute Inc., Cary, NC, USA).

\section{Results \\ Pre-screeners}

Among the 692 women pre-screened, 634 (91.6\%) were found to be eligible to continue with the in-depth screening. Approximately $97 \%$ of pre-screened women were willing to switch from their current contraceptive method to NuvaRing 
McLellan-Lemal et al. Research Journal of Women's Health 2016,

http://www.hoajonline.com/journals/pdf/2054-9865-3-1.pdf

doi: $10.7243 / 2054-9865-3-1$

for six months. As shown in Figure 1, the three most common reasons for pre-screening ineligibility were reluctance to switch to NuvaRing, not engaged in $>1$ episode of vaginal intercourse on different days in the past 30 days, and self-reported positive HIV status. Among the eligible pre-screened women, 26.9\% were screening visit no-shows. During re-contact attempts, some women told recruiters that they were concerned about partner support, discovery of the ring during sexual intercourse, and pain or discomfort associated with pelvic examinations.

\section{Screeners}

Out of 463 (73.0\%) women who completed the screening visit, three declined further study consideration. After meeting all eligibility criteria, 302 (99.3\%) of 304 women were enrolled into the pre-product phase of the study. Among women not eligible to take part in the study, reasons included testing positive for HIV (67/39.1\%), lack of OCP/DMPA documentation (39/22.8\%), body mass index $>29.0$ (34/19.9\%), currently breastfeeding or within three months of parturition (9/5.3\%), and laboratory confirmed pregnancy (8/4.7\%). Among enrollees, 54 (17.9\%) were OCPs users and 248 (82.1\%) were DMPA users.

Almost all (90.9\%) of the 463 screened were Luo, and $47.3 \%$ were between 18 and 24 years old, with a mean/median age of 25 (standard deviation 4.2) (Table 1). Approximately 63\% reported being employed, $55.7 \%$ reported salary-based earnings as a main source of income, $68.2 \%$ had a primary education or lower, $44.8 \%$ were Roman Catholic, and $67.9 \%$ were married or cohabitating. The mean number of live births was 2.5 (median 2.0; range: 0-8) with approximately $45 \%$ reporting that they had three or more live births.

As shown in Table 2, the three most common motivations for joining the study were to learn: about modern family planning methods (99.1\%), how to avoid HIV risk behaviors/behavior change (82.9\%), and about causes of HIV (79.5\%). In the bivari-

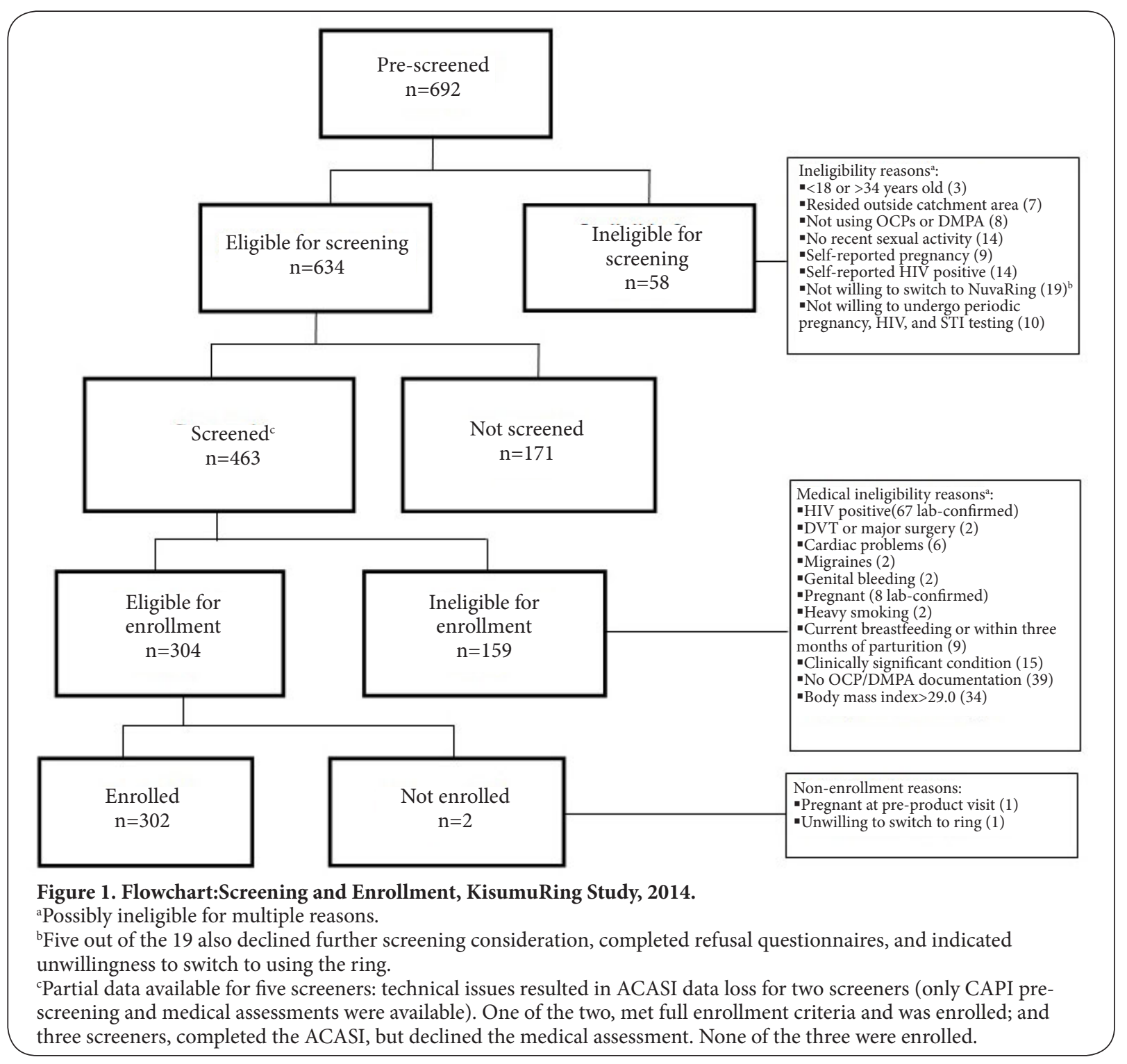


McLellan-Lemal et al. Research Journal of Women's Health 2016, http://www.hoajonline.com/journals/pdf/2054-9865-3-1.pdf

Table 1. Demographic Characteristics of Women Screened by Enrollment Status (n=463), Kisumu Ring Study, 2014.

\begin{tabular}{|c|c|c|c|c|c|c|}
\hline Variable Level & $\begin{array}{l}\text { Total } \\
(\mathrm{N}=463) \\
\mathrm{n}(\%)\end{array}$ & $\begin{array}{l}\text { Enrolled } \\
(\mathrm{N}=302) \\
\mathrm{n}(\%)\end{array}$ & $\begin{array}{l}\text { Not Enrolled } \\
(\mathrm{N}=161) \\
\mathrm{n}(\%)\end{array}$ & $\begin{array}{l}\text { Enrolled } \\
\text { Prevalence }\end{array}$ & $\begin{array}{l}\text { Prevalence } \\
\text { Ratio }(95 \% \text { CI })\end{array}$ & P-value \\
\hline Age at screening & & & & & & $0.003^{\star}$ \\
\hline $18-24$ & $219(47.3)$ & $159(52.6)$ & $60(37.3)$ & 72.6 & $1.47(1.15,1.88)$ & 0.002 \\
\hline $25-29$ & $169(36.5)$ & $106(35.1)$ & $63(39.1)$ & 62.7 & $1.27(0.98,1.64)$ & 0.067 \\
\hline $30-34$ & $75(16.2)$ & $37(12.3)$ & $38(23.6)$ & 49.3 & Ref. & -- \\
\hline Ethnic/tribal group & & & & & & 0.565 \\
\hline Luo & $418(90.9)$ & $271(90.3)$ & 147 (91.9) & 64.8 & $0.94(0.76,1.16)$ & 0.565 \\
\hline Non-Luo & $42(9.1)$ & $29(9.7)$ & $13(8.1)$ & 69.0 & Ref. & -- \\
\hline Marital status & & & & & & $0.001^{*}$ \\
\hline Single & $81(17.9)$ & $48(16.3)$ & $33(20.9)$ & 59.3 & $1.35(0.97,1.89)$ & 0.073 \\
\hline Married/cohabiting & 307 (67.9) & $218(74.1)$ & $89(56.3)$ & 71.0 & $1.62(1.22,2.16)$ & 0.001 \\
\hline Separated/divorced/widowed & $64(14.2)$ & $28(9.5)$ & $36(22.8)$ & 43.8 & Ref. & -- \\
\hline Religion & & & & & & 0.989 \\
\hline Roman Catholic & $206(44.8)$ & $135(45.0)$ & $71(44.4)$ & 65.5 & $1.01(0.84,1.22)$ & 0.886 \\
\hline Other Christian & $172(37.4)$ & $112(37.3)$ & $60(37.5)$ & 65.1 & $1.01(0.83,1.22)$ & 0.940 \\
\hline Other non-Christian & $82(17.8)$ & $53(17.7)$ & $29(18.1)$ & 64.6 & Ref. & -- \\
\hline Highest education completed & & & & & & 0.817 \\
\hline Primary or less ${ }^{\mathrm{a}}$ & $313(68.2)$ & $205(68.6)$ & $108(67.5)$ & 65.5 & $0.97(0.74,1.26)$ & 0.817 \\
\hline Secondary or higher & $146(31.8)$ & $94(31.4)$ & $52(32.5)$ & 64.4 & Ref. & -- \\
\hline Employment status & & & & & & 0.346 \\
\hline Employed & $291(63.4)$ & $185(61.9)$ & $106(66.3)$ & 63.6 & $0.94(0.82,1.07)$ & 0.346 \\
\hline Unemployed & $168(36.6)$ & $114(38.1)$ & $54(33.8)$ & 67.9 & Ref. & -- \\
\hline Main source of personal income & & & & & & $0.026^{*}$ \\
\hline None & $22(4.8)$ & $12(4.0)$ & $10(6.3)$ & 54.5 & $0.75(0.51,1.11)$ & 0.154 \\
\hline Salary-based & $256(55.7)$ & $157(52.2)$ & $99(62.3)$ & 61.3 & $0.85(0.74,0.97)$ & 0.013 \\
\hline Not salary-based & $182(39.6)$ & $132(43.9)$ & $50(31.4)$ & 72.5 & Ref. & -- \\
\hline Number of live births ${ }^{b}$ & & & & & & 0.433 \\
\hline $0-2$ & $245(54.6)$ & $166(55.9)$ & $79(52.0)$ & 67.8 & $1.60(0.92,1.21)$ & 0.433 \\
\hline $3+$ & $204(45.4)$ & $131(44.1)$ & $73(48.0)$ & 64.2 & Ref. & -- \\
\hline
\end{tabular}

Sample sizes fluctuate slightly for some variables due to missing data. Some percentages do not sum to 100 because of rounding.

${ }^{\mathrm{a}} 17$ reported no schooling; 136 reported attending, but not completing primary education; 160 completed primary education.

b51 reported one or more miscarriages/abortions (range: 1-7); and 6 reported still births (range: 1-2).

${ }^{*}$ Variable met $\mathrm{p}$-value $\leq .20$ criteria for inclusion in the multivariable analysis.

ate analysis, women who did not report free medical care for STIs as a motivator were less likely to be enrolled (prevalence ratio $[\mathrm{PR}]=0.84,95 \%$ confidence interval $[\mathrm{Cl}]=0.73-0.97$ ) than those enrolled. Women who reported wanting to learn how to avoid HIV risk behaviors as a motivator were less likely to be enrolled (PR 0.85, 95\% Cl 0.73-0.99) than those enrolled. Regardless of enrollment status, receiving incentives was the least common motivator overall, with $31 \%$ of women screened reporting that they were interested in joining the study for this reason.

Overall, less than $10 \%$ reported that they had desired or wanted to get pregnant within the next 12 months: 7.0\% indicated that they wanted to be pregnant in the next 12 months and $9.6 \%$ planned to get pregnant in the next 12 months. Slightly over $13 \%$ responded that their partner wanted them to get pregnant in the next 12 months. Few barriers to using modern contraceptives were identified. Barriers predominately centered on concerns regarding access (17.6\%), affordability (15.5\%), and side effects (15.0\%). Approximately a quarter $(25.2 \%)$ reported that they had used two or more birth control methods over the past 12 months (Table 3). No significant difference in acceptance of pelvic exams was observed between women enrolled and women not enrolled. Overall, 51.3\% scored medium acceptance of pelvic examinations.

Most of the women (90.8\%) reported ever being pregnant (Table 3). Overall, prevalence was $1.7 \%$ for pregnancy, $14.5 \%$ for HIV, and $70.4 \%$ for other STIs. Sexual debut before the age 
Table 2. Psychosocial Characteristics of Women Screened by Enrollment Status ( $n=463)$, Kisumu Ring Study, 2014.

\begin{tabular}{|c|c|c|c|c|c|c|}
\hline Variable Level & $\begin{array}{l}\text { Total } \\
(\mathrm{N}=463) \\
\mathrm{n}(\%) \\
\end{array}$ & $\begin{array}{l}\text { Enrolled } \\
(\mathrm{N}=302) \\
\mathrm{n}(\%) \\
\end{array}$ & $\begin{array}{l}\text { Not Enrolled } \\
(\mathrm{N}=161) \\
\mathrm{n}(\%)\end{array}$ & $\begin{array}{l}\text { Enrolled } \\
\text { Prevalence }\end{array}$ & $\begin{array}{l}\text { Prevalence } \\
\text { Ratio }(95 \% \mathrm{CI})\end{array}$ & P-value \\
\hline \multicolumn{7}{|l|}{ Motivations for Participation: } \\
\hline Receive free HIV testing and counseling & & & & & & 0.934 \\
\hline Yes & $316(69.0)$ & $206(69.1)$ & $110(68.8)$ & 65.2 & $1.01(0.87,1.16)$ & 0.934 \\
\hline No & $142(31.0)$ & $92(30.9)$ & $50(31.3)$ & 64.8 & Ref. & -- \\
\hline $\begin{array}{l}\text { Receive free medical care for STIs and common } \\
\text { illnesses }\end{array}$ & & & & & & $0.015^{*}$ \\
\hline Yes & $356(77.9)$ & $222(74.7)$ & $134(83.8)$ & 62.4 & $0.84(0.73,0.97)$ & 0.015 \\
\hline No & $101(22.1)$ & $75(25.3)$ & $26(16.3)$ & 74.3 & Ref. & -- \\
\hline Receive incentives & & & & & & 0.328 \\
\hline Yes & $141(31.1)$ & $96(32.7)$ & $45(28.3)$ & 68.1 & $0.93(0.79,1.08)$ & 0.328 \\
\hline No & $312(68.9)$ & $198(67.3)$ & $114(71.7)$ & 63.5 & Ref. & -- \\
\hline Learn about causes of HIV & & & & & & 0.330 \\
\hline Yes & $364(79.5)$ & $233(78.2)$ & $131(81.9)$ & 64.0 & $1.17(0.84,1.63)$ & 0.330 \\
\hline No & $94(20.5)$ & $65(21.8)$ & $29(18.1)$ & 69.1 & Ref. & -- \\
\hline $\begin{array}{l}\text { Be able to share with others information learned } \\
\text { about HIV }\end{array}$ & & & & & & $0.134^{\star}$ \\
\hline Yes & $314(68.9)$ & $197(66.6)$ & $117(73.1)$ & 62.7 & $0.90(0.78,1.00)$ & 0.134 \\
\hline No & $142(31.1)$ & $99(33.4)$ & $43(26.9)$ & 69.7 & Ref. & -- \\
\hline Help in controlling the spread of HIV & & & & & & $0.158^{\star}$ \\
\hline Yes & $359(78.4)$ & $228(76.5)$ & $131(81.9)$ & 63.5 & $0.90(0.77,1.04)$ & 0.158 \\
\hline No & $99(21.6)$ & $70(23.5)$ & $29(18.1)$ & 70.7 & Ref. & -- \\
\hline Learn how to avoid HIV risk behaviors & & & & & & $\mathbf{0 . 0 3 6}^{*}$ \\
\hline Yes & $378(82.9)$ & $239(80.5)$ & $139(87.4)$ & 63.2 & $0.85(0.73,0.99)$ & 0.036 \\
\hline No & $78(17.1)$ & $58(19.5)$ & $20(12.6)$ & 74.4 & Ref. & -- \\
\hline \multicolumn{7}{|l|}{ Learn about modern family planning methods } \\
\hline Yes & $454(99.1)$ & $294(98.7)$ & $160(100.0)$ & 64.8 & -- & -- \\
\hline No & $4(0.9)$ & $4(1.3)$ & $0(0.0)$ & 100.0 & Ref. & -- \\
\hline \multicolumn{7}{|l|}{ Pregnancy Desires/Intentions } \\
\hline Pregnancy desired $t$ in the next 12 months & & & & & & 0.747 \\
\hline Yes & $32(7.0)$ & $20(6.7)$ & $12(7.5)$ & 62.5 & $0.96(0.72,1.26)$ & 0.747 \\
\hline No & $425(93.0)$ & $278(93.3)$ & $147(92.5)$ & 65.4 & Ref. & -- \\
\hline Pregnancy planned in the next 12 months & & & & & & 0.396 \\
\hline Yes & $44(9.6)$ & $31(10.4)$ & $13(8.1)$ & 70.5 & $1.09(0.89,1.34)$ & 0.396 \\
\hline No & $414(90.4)$ & $267(89.6)$ & $147(91.9)$ & 64.5 & Ref. & -- \\
\hline Partner wants her pregnant in the next 12 months & & & & & & $\mathbf{0 . 1 3 3}^{*}$ \\
\hline Yes & $59(13.1)$ & $43(14.7)$ & $16(10.2)$ & 72.9 & $1.14(0.96,1.36)$ & 0.133 \\
\hline No & $390(86.9)$ & $249(85.3)$ & $141(89.8)$ & 63.8 & Ref. & -- \\
\hline \multicolumn{7}{|l|}{ Barriers to Modern Contraceptive Method Use } \\
\hline Too difficult to get & & & & & & 0.230 \\
\hline Yes & $76(17.6)$ & $45(15.8)$ & $31(20.8)$ & 59.2 & $0.88(0.72,1.08)$ & 0.230 \\
\hline No & $357(82.4)$ & $239(84.2)$ & $118(79.2)$ & 66.9 & Ref. & -- \\
\hline Unable to afford & & & & & & 0.230 \\
\hline Yes & $67(15.5)$ & $39(13.7)$ & $28(18.8)$ & 58.2 & $0.87(0.70,1.08)$ & 0.230 \\
\hline No & $366(84.5)$ & $245(86.3)$ & $121(81.2)$ & 66.9 & Ref. & -- \\
\hline Unsure how to use desired method & & & & & & 0.811 \\
\hline Yes & $20(4.6)$ & $14(4.9)$ & $6(4.0)$ & 70.0 & -- & -- \\
\hline No & $413(95.4)$ & $270(95.1)$ & $143(96.0)$ & 65.4 & Ref. & -- \\
\hline Afraid of side or bad effects & & & & & & 0.653 \\
\hline Yes & $65(15.0)$ & $41(14.4)$ & $24(16.1)$ & 63.1 & $0.96(0.78,1.17)$ & 0.653 \\
\hline No & $368(85.0)$ & $243(85.6)$ & $125(83.9)$ & 66.0 & Ref. & -- \\
\hline
\end{tabular}


Continuation of Table 2.

\begin{tabular}{|c|c|c|c|c|c|c|}
\hline Variable Level & $\begin{array}{l}\text { Total } \\
(\mathrm{N}=463) \\
\mathrm{n}(\%)\end{array}$ & $\begin{array}{l}\text { Enrolled } \\
(\mathrm{N}=302) \\
\mathrm{n}(\%) \\
\end{array}$ & $\begin{array}{l}\text { Not Enrolled } \\
(\mathrm{N}=161) \\
\mathrm{n}(\%) \\
\end{array}$ & $\begin{array}{l}\text { Enrolled } \\
\text { Prevalence }\end{array}$ & $\begin{array}{l}\text { Prevalence } \\
\text { Ratio }(95 \% \text { CI })\end{array}$ & P-value \\
\hline Partner will/might disapprove & & & & & & 0.592 \\
\hline Yes & $36(8.3)$ & $25(8.8)$ & $11(7.4)$ & 69.4 & $1.06(0.85,1.34)$ & 0.592 \\
\hline No & $397(91.7)$ & $259(91.2)$ & $138(92.6)$ & 65.2 & Ref. & -- \\
\hline Community will/might disapprove & & & & & & 0.548 \\
\hline Yes & $2(0.5)$ & $2(0.7)$ & $0(0.0)$ & 100.0 & -- & -- \\
\hline No & $431(99.5)$ & $282(99.3)$ & $149(100.0)$ & 65.4 & Ref. & \\
\hline Church/religious community might disapprove & & & & & & $0.170^{*}$ \\
\hline Yes & $5(1.2)$ & $5(1.8)$ & $0(0.0)$ & 100.0 & -- & -- \\
\hline No & $428(98.8)$ & $279(98.2)$ & $149(100.0)$ & 65.2 & Ref. & -- \\
\hline Wants children & & & & & & 0.664 \\
\hline Yes & $5(1.2)$ & $4(1.4)$ & $1(0.7)$ & 80.0 & -- & -- \\
\hline No & $428(98.8)$ & $280(98.6)$ & $148(99.3)$ & 65.4 & Ref. & -- \\
\hline Other barriers & & & & & & 1.000 \\
\hline Yes & $5(1.2)$ & $3(1.1)$ & $2(1.3)$ & 60.0 & -- & -- \\
\hline No & $428(98.8)$ & $281(98.9)$ & $147(98.7)$ & 65.7 & -- & -- \\
\hline None, nothing can prevent use & & & & & & 0.878 \\
\hline Yes & $178(41.1)$ & $116(40.8)$ & $62(41.6)$ & 65.2 & $0.99(1.14,1.33)$ & 0.878 \\
\hline No & $255(58.9)$ & $168(59.2)$ & $87(58.4)$ & 65.9 & Ref. & -- \\
\hline \multicolumn{7}{|l|}{ Study ProceduresAcceptance } \\
\hline \multicolumn{7}{|c|}{ Switch to NuvaRing for 6 months (notional acceptability) } \\
\hline Yes & $463(100.0)$ & $302(100.0)$ & $161(100.0)$ & 100.0 & -- & -- \\
\hline \multicolumn{7}{|l|}{ Undergo periodic testing for pregnancy, HIV and STIs } \\
\hline Yes & $463(100.0)$ & $302(100.0)$ & $161(100.0)$ & 100.0 & -- & -- \\
\hline Routine pelvic exam & & & & & & 0.855 \\
\hline High & $142(31.0)$ & $95(31.9)$ & $47(29.4)$ & 66.9 & $1.04(0.85,1.27)$ & 0.685 \\
\hline Medium & $235(51.3)$ & $151(50.7)$ & $84(52.5)$ & 64.3 & $1.00(0.83,1.21)$ & 0.993 \\
\hline Low & $81(17.7)$ & $52(17.4)$ & $29(18.1)$ & 64.2 & Ref. & -- \\
\hline
\end{tabular}

Sample sizes fluctuate slightly for some variables due to missing data. Some percentages do not sum to 100 because of rounding.

${ }^{*}$ Variable met $\mathrm{p}$-value $\leq .20$ criteria for inclusion in the multivariable analysis.

of 17 was reported by $54.8 \%$. While no statistical differences were observed between those enrolled and not enrolled, $34.6 \%$ of women screened reported experiencing physically forced sex at some point in their lives. Women who reported a single lifetime sexual partner (PR 1.34, 95\% CI 1.07-1.67) or those reporting 2-3 lifetime sexual partners $(P R=1.23,95 \%$ $\mathrm{Cl} 1.02-1.59)$ were more likely to be enrolled than those who reported four or more lifetime partners Women who reported a single sexual partner in the past three months (PR 1.42, 95\% $\mathrm{Cl}$ 1.07-1.88) were more likely to be enrolled than those who reported two or more sexual partners in the past 3 months. While data were collected separately for vaginal and anal sex in the past 12 months, we combined these variables given that the prevalence for anal intercourse in the past 12 months for all women who completed the screening ACASI was $7.3 \%$. Incidentally, 33 out of 34 women reporting anal intercourse in the past 12 months reported that condoms were not used. Overall, $91.9 \%$ engaged in vaginal or anal sex without a condom in the past three months. Women using
DMPA in the past 12 months were more likely to be enrolled ( $P R=1.36,95 \% \mathrm{Cl} 1.09-1.69)$.

In the multivariable model, enrollment was significantly $(p<0.05)$ more likely among women who were aged 18-24 years old, married/cohabitating, reported sexual debut at less than 17 years of age, had one lifetime sexual partner, abnormal vaginal bleeding in the past 12 months, vaginal or anal sex without a condom in the past three months, and did not have a sexual partner of unknown HIV status in the past three months. DMPA use in the past 12 months was not significant in the multivariable model (Table 4).

\section{Discussion}

This study successfully recruited and enrolled women for the pre-product use phase of a contraceptive IVR study in Kisumu, Kenya. Approximately for every five women pre-screened, two were enrolled in our study. Multivariable regression analysis showed that enrollment was significantly higher among women who: were less than 25 years of age, reported 
Table 3. Behavioral Characteristics of Women Screened by Enrollment Status (n=463), Kisumu Ring Study, 2014.

\begin{tabular}{|c|c|c|c|c|c|c|}
\hline Variable Level & $\begin{array}{l}\text { Total } \\
(\mathrm{N}=463) \\
\mathrm{n}(\%) \\
\end{array}$ & $\begin{array}{l}\text { Enrolled } \\
(\mathrm{N}=302) \\
\mathrm{n}(\%) \\
\end{array}$ & $\begin{array}{l}\text { Not Enrolled } \\
(\mathrm{N}=161) \\
\mathrm{n}(\%) \\
\end{array}$ & $\begin{array}{l}\text { Enrolled } \\
\text { Prevalence }\end{array}$ & $\begin{array}{l}\text { Prevalence } \\
\text { Ratio }(95 \% \mathrm{CI})\end{array}$ & P-value \\
\hline \multicolumn{7}{|l|}{ Reproductive and Sexual Health } \\
\hline Age at sexual debut ${ }^{\mathrm{a}}$ & & & & & & 0.328 \\
\hline 13 or less & $77(20.8)$ & $50(21.0)$ & $27(20.3)$ & 64.9 & $1.33(0.93,1.91)$ & 0.119 \\
\hline $14-16$ & $126(34.0)$ & $85(35.7)$ & $41(30.8)$ & 67.5 & $1.38(0.98,1.95)$ & 0.064 \\
\hline $17-19$ & $129(34.8)$ & $84(35.3)$ & $45(33.8)$ & 65.1 & $1.34(0.95,1.89)$ & 0.100 \\
\hline $20+$ & $39(10.5)$ & $19(8.0)$ & $20(15.0)$ & 48.7 & Ref. & -- \\
\hline Ever experienced physically forced sex & & & & & & 0.446 \\
\hline Yes & $121(34.6)$ & $82(36.0)$ & $39(32.0)$ & 67.8 & $1.06(0.91,1.24)$ & 0.446 \\
\hline No & $229(65.4)$ & $146(64.0)$ & $83(68.0)$ & 63.8 & Ref. & -- \\
\hline \# lifetime sexual partners & & & & & & $0.029^{\star}$ \\
\hline 1 & $60(15.9)$ & $44(18.1)$ & $16(11.9)$ & 73.3 & $1.34(1.07,1.67)$ & 0.011 \\
\hline $2-3$ & $195(51.7)$ & $132(54.3)$ & $63(47.0)$ & 67.7 & $1.23(1.02,1.59)$ & 0.029 \\
\hline $4+$ & $122(32.4)$ & $67(27.6)$ & $55(41.0)$ & 54.9 & Ref. & -- \\
\hline \# partners in past 3 months & & & & & & $0.016^{\star}$ \\
\hline 1 & $272(82.9)$ & $186(87.3)$ & $86(74.8)$ & 68.4 & $1.42(1.07,1.88)$ & 0.016 \\
\hline $2+$ & $56(17.1)$ & $27(12.7)$ & $29(25.2)$ & 48.2 & Ref. & \\
\hline $\mathrm{HIV}+$ partner in the past 3 months & & & & & & 0.483 \\
\hline Yes & $83(27.7)$ & $51(26.3)$ & $32(30.2)$ & 61.4 & $0.93(0.77,1.13)$ & 0.483 \\
\hline No & $217(72.3)$ & $143(73.7)$ & $74(69.8)$ & 65.9 & Ref. & -- \\
\hline $\begin{array}{l}\text { HIV status unknown partnersin past } \\
3 \text { months }\end{array}$ & & & & & & $0.118^{\star}$ \\
\hline Yes & $153(50.5)$ & $94(47.2)$ & $59(56.7)$ & 61.4 & $0.88(0.75,1.03)$ & 0.118 \\
\hline No & $150(49.5)$ & $105(52.8)$ & $45(43.3)$ & 70.0 & Ref. & -- \\
\hline $\begin{array}{l}\text { Abnormal vaginal bleeding in the past } \\
12 \text { months }\end{array}$ & & & & & & $0.020^{\star}$ \\
\hline Yes & $100(21.8)$ & $74(24.8)$ & $26(16.3)$ & 74.0 & $1.18(1.03,1.36)$ & 0.020 \\
\hline No & $358(78.2)$ & $224(75.2)$ & $134(83.8)$ & 62.6 & Ref. & -- \\
\hline Exchange $\operatorname{sex}^{\mathrm{b}}$ in the past 3 months & & & & & & 0.923 \\
\hline Yes & $48(14.0)$ & $31(13.9)$ & $17(14.3)$ & 64.6 & $0.99(0.79,1.24)$ & 0.923 \\
\hline No & $294(86.0)$ & $192(86.1)$ & $102(85.7)$ & 65.3 & Ref. & -- \\
\hline $\begin{array}{l}\text { Vaginal or anal sex }{ }^{\mathrm{c}} \text { without a condom } \\
\text { in the past } 3 \text { months }\end{array}$ & & & & & & $0.197^{\star}$ \\
\hline Yes & $305(91.9)$ & $202(93.5)$ & $103(88.8)$ & 66.2 & $1.28(0.88,1.85)$ & 0.197 \\
\hline No & $27(8.1)$ & $14(6.5)$ & $13(11.2)$ & 51.9 & Ref. & -- \\
\hline Ever been pregnant & & & & & & 0.460 \\
\hline Yes & $416(90.8)$ & $273(91.6)$ & $143(89.4)$ & 65.6 & $1.10(0.85,1.43)$ & 0.460 \\
\hline No & $42(9.2)$ & $25(8.4)$ & $17(10.6)$ & 59.5 & Ref. & -- \\
\hline Currently pregnant (self-report) & & & & & & 0.698 \\
\hline Yes & $7(1.5)$ & $4(1.3)$ & $3(1.9)$ & 57.1 & -- & -- \\
\hline No & $450(98.5)$ & $294(98.7)$ & $156(98.1)$ & 65.3 & Ref. & -- \\
\hline Pregnancy test result & & & & & & $<0.001^{\star}$ \\
\hline Positive & $8(1.7)$ & $0(0.0)$ & $8(5.0)$ & 0.0 & -- & -- \\
\hline Negative/Other & $453(98.3)$ & $302(100.0)$ & $151(95.0)$ & 66.7 & Ref. & -- \\
\hline $\begin{array}{l}\text { Number contraceptive methods past } \\
12 \text { months }\end{array}$ & & & & & & $0.005^{\star}$ \\
\hline 0 & $11(2.4)$ & $2(0.7)$ & $9(5.6)$ & 18.2 & -- & -- \\
\hline 1 & $334(72.5)$ & $223(74.1)$ & $111(69.4)$ & 66.8 & -- & -- \\
\hline $2+$ & $116(25.2)$ & $76(25.2)$ & $40(25.0)$ & 65.5 & Ref. & -- \\
\hline
\end{tabular}


McLellan-Lemal et al. Research Journal of Women's Health 2016, http://www.hoajonline.com/journals/pdf/2054-9865-3-1.pdf

Continuation of Table 3.

\begin{tabular}{|c|c|c|c|c|c|c|}
\hline Variable Level & $\begin{array}{l}\text { Total } \\
(\mathrm{N}=463) \\
\mathrm{n}(\%)\end{array}$ & $\begin{array}{l}\text { Enrolled } \\
(\mathrm{N}=302) \\
\mathrm{n}(\%)\end{array}$ & $\begin{array}{l}\text { Not Enrolled } \\
(\mathrm{N}=161) \\
\mathrm{n}(\%)\end{array}$ & $\begin{array}{l}\text { Enrolled } \\
\text { Prevalence }\end{array}$ & $\begin{array}{l}\text { Prevalence } \\
\text { Ratio }(95 \% \text { CI })\end{array}$ & P-value \\
\hline Ever used female condom & & & & & & $0.192^{\star}$ \\
\hline Yes & $55(12.0)$ & $31(10.4)$ & $24(15.0)$ & 56.4 & $0.85(0.67,1.08)$ & 0.192 \\
\hline No & $403(88.0)$ & $267(89.6)$ & $136(85.0)$ & 66.3 & Ref. & -- \\
\hline Ever had sex during menses & & & & & & 0.256 \\
\hline Not applicable & $65(14.1)$ & $39(13.0)$ & $26(16.3)$ & 60.0 & $0.84(0.67,1.06)$ & 0.149 \\
\hline No & $294(63.9)$ & $189(63.0)$ & $105(65.6)$ & 64.3 & $0.90(0.78,1.05)$ & 0.178 \\
\hline Yes & $101(22.0)$ & $72(24.0)$ & $29(18.1)$ & 71.3 & Ref. & -- \\
\hline Ever had a STI (self-reported) & & & & & & 0.574 \\
\hline Yes & $109(29.1)$ & $74(30.1)$ & $35(27.3)$ & 67.9 & $1.05(0.89,1.22)$ & 0.574 \\
\hline No & $265(70.9)$ & $172(69.9)$ & $93(72.7)$ & 64.9 & Ref. & -- \\
\hline$\geq 1$ STI test results ${ }^{d}$ & & & & & & $0.197^{\star}$ \\
\hline Yes & $321(70.4)$ & $204(68.5)$ & $117(74.1)$ & 63.6 & $0.91(0.79,1.05)$ & 0.197 \\
\hline No & $135(29.6)$ & $94(31.5)$ & $41(25.9)$ & 69.6 & Ref. & -- \\
\hline HIV test result & & & & & & $<\mathbf{0 . 0 0 1}^{\star}$ \\
\hline Positive & $67(14.5)$ & $0(0.0)$ & $67(42.1)$ & 0.0 & -- & -- \\
\hline Negative & $394(85.5)$ & $302(100.0)$ & $92(57.9)$ & 76.6 & Ref. & -- \\
\hline $\begin{array}{l}\text { Past medication adherence history } \\
\text { (lifetime) }\end{array}$ & & & & & & 0.570 \\
\hline High & $166(36.4)$ & $111(37.2)$ & $55(34.8)$ & 66.9 & $1.10(0.91,1.31)$ & 0.328 \\
\hline Modest & $177(38.8)$ & $118(39.6)$ & $59(37.3)$ & 66.7 & $1.09(0.91,1.31)$ & 0.340 \\
\hline Low & $113(24.8)$ & $69(23.2)$ & $44(27.8)$ & 61.1 & Ref. & -- \\
\hline \multicolumn{7}{|l|}{ Substance use } \\
\hline Alcohol use in past 30 days & & & & & & 0.239 \\
\hline Yes & $111(24.0)$ & $67(22.2)$ & $44(27.3)$ & 60.4 & $0.90(0.76,1.07)$ & 0.239 \\
\hline No & $352(76.0)$ & $235(77.8)$ & $117(72.7)$ & 66.8 & Ref. & -- \\
\hline Ever used drugs & & & & & & 0.825 \\
\hline Yes & $27(5.9)$ & $17(5.7)$ & $10(6.3)$ & 63.0 & $0.97(0.72,1.30)$ & 0.825 \\
\hline No & $430(94.1)$ & $280(94.3)$ & $150(93.8)$ & 65.1 & Ref. & -- \\
\hline
\end{tabular}

Sample sizes fluctuate slightly for some variables due to missing data. Some percentages do not sum to 100 because of rounding. *Variable met p-value $\leq .20$ criteria for inclusion in the multivariable analysis.

a22.9\% reported first sex before the age of 15 .

bReceived gifts, money, food, shelter, cosmetics, other material goods, and/or services for providing sex.

c 34 women reported anal sex in the past 12 months; 33 out of the 34 indicated that a condom was not used.

d54.9\% tested positive for HSV-2, 3.9\% for gonorrhea, 1.9\% for syphilis, $4.5 \%$ for chlamydia, and 38.0\% for bacterial vaginosis.

a single lifetime sexual partner, did not have a recent partner of unknown HIV status, had experienced sexual debut before the age of 17 , and had abnormal vaginal bleeding in the past 12 months.

Only about 1 out of 4 women uses a modern contraceptive method in sub-Saharan Africa [18]. Reproductive age accounts for some differences in contraceptive method choice and motivations for use. Data collected between 2004 and 2010 in 18 sub-Saharan African countries showed that the use of modern contraceptives to limit births was highest among women 35 years of age and older, while contraceptive use to space births was characteristic of women 25-29 years of age [18]. Younger women in our study may have been more interested in trying new technologies, especially short-term methods to space births. Cultural expectations for young mar- ried women to have children sooner rather than later [19] as well as beliefs regarding "having the right number of children" [20] could influence method choice, especially preferences that minimize detection of use by others or lessen inabilities to conceive when use of a method has stopped.

Early initiation of sexual intercourse (marital as well as premarital) among women has been shown to be associated with either low [21] or erratic [22] contraceptive use, including lower condom use to protect against HIV and other STIs. In our study, women with an age of sexual debut less than 17 years may have been more interested in taking part in the study because they had probably already experienced at least one pregnancy and were either using OCPs or DMPA. Since we did not enroll contraceptive-naïve women, it is unknown if their interest in an IVR would differ. 
McLellan-Lemal et al. Research Journal of Women's Health 2016,

http://www.hoajonline.com/journals/pdf/2054-9865-3-1.pdf

doi: 10.7243/2054-9865-3-1

Table 4. Final Multivariable Model for Women Screened by Enrollment Status (n=289), Kisumu Ring Study, 2014.

\begin{tabular}{|c|c|c|c|c|c|c|}
\hline Variable Level & $\begin{array}{l}\text { Total } \\
(\mathrm{N}=289) \\
\mathrm{n}(\%)\end{array}$ & $\begin{array}{l}\text { Enrolled } \\
(\mathrm{N}=188) \\
\mathrm{n}(\%) \\
\end{array}$ & $\begin{array}{l}\text { Not Enrolled } \\
(\mathrm{N}=101) \\
\mathrm{n}(\%) \\
\end{array}$ & $\begin{array}{l}\text { Prevalence } \\
\text { of Enrolled }\end{array}$ & $\begin{array}{l}\text { Adj. Prevalence } \\
\text { Ratio (95\% CI) }\end{array}$ & P-value \\
\hline Age at screening & & & & & & 0.001 \\
\hline $18-24$ & $147(50.9)$ & $111(59.0)$ & $36(35.6)$ & 75.5 & $1.59(1.16,2.16)$ & 0.003 \\
\hline $25-29$ & $94(32.5)$ & $56(29.8)$ & $38(37.6)$ & 59.6 & $1.24(0.89,1.73)$ & 0.205 \\
\hline $30-34$ & $48(16.6)$ & $21(11.2)$ & $27(26.7)$ & 43.8 & Ref. & -- \\
\hline Marital status & & & & & & 0.002 \\
\hline Single & $56(19.4)$ & $32(17.0)$ & $24(23.8)$ & 57.1 & $1.11(0.75,1.64)$ & 0.610 \\
\hline Married/cohabiting & $190(65.7)$ & $138(73.4)$ & $52(51.5)$ & 72.6 & $1.52(1.08,2.14)$ & 0.016 \\
\hline Separated/divorced/widowed & $43(14.9)$ & $18(9.6)$ & $25(24.8)$ & 41.9 & Ref. & -- \\
\hline $\begin{array}{l}\text { Had abnormal vaginal bleeding in the past } \\
12 \text { months }\end{array}$ & & & & & & 0.036 \\
\hline Yes & $59(20.4)$ & $47(25.0)$ & $12(11.9)$ & 79.7 & $1.21(1.01,1.44)$ & 0.036 \\
\hline No & $230(79.6)$ & $141(75.0)$ & $89(88.1)$ & 61.3 & Ref. & -- \\
\hline Age at sexual debut & & & & & & 0.067 \\
\hline 13 or younger & $57(19.7)$ & $42(22.3)$ & $15(14.9)$ & 73.7 & $1.58(1.10,2.27)$ & 0.013 \\
\hline $14-16$ & $100(34.6)$ & $69(36.7)$ & $31(30.7)$ & 69.0 & $1.56(1.09,2.22)$ & 0.015 \\
\hline $17-19$ & $98(33.9)$ & $62(33.0)$ & $36(35.6)$ & 63.3 & $1.38(0.97,1.97)$ & 0.074 \\
\hline $20+$ & $34(11.8)$ & $15(8.0)$ & $19(18.8)$ & 44.1 & Ref. & -- \\
\hline \# lifetime sexual partners & & & & & & 0.040 \\
\hline 1 & $44(15.2)$ & $34(18.1)$ & $10(9.9)$ & 77.3 & $1.36(1.07,1.72)$ & 0.012 \\
\hline $2-3$ & $152(52.6)$ & $105(55.9)$ & $47(46.5)$ & 69.1 & $1.21(0.99,1.48)$ & 0.064 \\
\hline $4+$ & $93(32.2)$ & $49(26.1)$ & $44(43.6)$ & 52.7 & Ref. & -- \\
\hline $\begin{array}{l}\text { Vaginal or anal sex without a condom in the } \\
\text { past } 3 \text { months }\end{array}$ & & & & & & 0.046 \\
\hline Yes & $265(91.7)$ & $176(93.6)$ & $89(88.1)$ & 66.4 & $1.43(1.01,2.02)$ & 0.046 \\
\hline No & $24(8.3)$ & $12(6.4)$ & $12(11.9)$ & 50.0 & Ref. & -- \\
\hline $\begin{array}{l}\text { HIV status unknown partners in past } 3 \\
\text { months }\end{array}$ & & & & & & 0.017 \\
\hline Yes & $144(49.8)$ & $86(45.7)$ & $58(57.4)$ & 59.7 & $0.82(0.70,0.97)$ & 0.017 \\
\hline No & $145(50.2)$ & $102(54.3)$ & $43(42.6)$ & 70.3 & Ref. & -- \\
\hline
\end{tabular}

While women with one lifetime sexual partner and those who did not have a recent partner of unknown HIV status were at lower risk for HIV, their risk for unintended pregnancy and possibly unsafe abortions is unknown. The literature shows that women are more likely to forgo condom use given concerns about intimacy and trust with a main partner [23]. A recent qualitative study found that condoms were not considered as contraception by young Kenyan women [24]. Moreover, the perception that contraceptive use, including condom use, contribute to disease, promiscuity, and infidelity has been suggested in several studies [24-26].

Studies on the optimal ratio of women enrolled to those screened for IVRs and other contraceptive technologies are sparse; thus, making it somewhat difficult to ascertain if our enrollment to screening ratio (ESR) was high or low. In Cameroon, a preventive HIV/STI trial of a vaginally inserted nonoxynol 9 showed a 57.5\% ESR (1317 enrolled among 2290 screened) [27]. The US-based Contraceptive CHOICE Project, which examined choice of free reversible contraceptive, suggested a $60.9 \%$ ESR ( 2500 enrolled out of 4107 screened) [28]. While the ESR is somewhat higher in these other studies, important contextual factors need to be taken into account for our sample (e.g., novelty of IVR, modern contraceptive use prevalence, potential inability to keep partner from knowing about IVR use). In addition, it is possible that our eligibility screening criteria may have affected our ESR by excluding women who were HIV-infected or unable to provide documentation of DMAP or OCP use.

While high pre-use, information-only-based acceptance of an IVR is suggested, caution must be taken in interpreting our findings, especially given that willingness to switch to Nuva Ring was a study eligibility criterion. At most, our findings may suggest that the availability of a new contraceptive option was appealing to women in our sample. This is further supported by results that showed that learning about modern family planning was the most common motivator for seeking study participation. In addition, concerns with abnormal vaginal bleeding in the past 12 months that may have been associ- 
ated with the contraceptive method reported at screening, especially DMPA [28], may have influenced women's willingness to try a new method. We acknowledge that the NuvaRing information provided during the screening process, while thorough, does not provide sufficient insights on readiness and acceptance. The concept of acceptability consists of two components: (a) willingness, which gets at mental readiness or inclination to try a product in the future or to recommend its use to others, and (b) use, which transforms intentions into actual experience that usually involves following prescribed instructions for correct and consistent use of a product or product substitute [29]. Women pre-screened for our study reported high NuvaRing notional acceptability. An accurate assessment of contraceptive IVR acceptability will be dependent on completion of all phases of the study.

We observed high prevalence of HIV, HSV-2, and BV. The Government of Kenya has identified Kisumu as one of the top three counties with a hyper-endemic HIV burden, with prevalence among women slightly higher than that of all of Kenya ( $20.3 \%$ versus $19.3 \%$, respectively) and the median age of HIV acquisition significantly younger among women than men [30]. The literature shows that HSV-2 and BV are significantly associated with a risk for acquiring HIV [31], that HSV-2 increases the risk for BV [32], and that prevalent and incident HSV-2 infection is linked to an increased prevalence of BV [33-35]. A comprehensive approach to women's sexual and reproductive health would be of benefit in this setting.

We found a slightly higher percentage of women who reported sexual debut before the age of 15 than was reported in the 2011 Nyanza Province Multiple Indicator Cluster Survey ( $22.9 \%$ vs. $18.9 \%)$, which may be attributed to our suvery administration mode (ACASI vs. face-to-face intervieweradministered survey) or the age of our participants (18-34 years vs. 15-24 years) [36]. The evidence linking early sexual debut and lifetime risk for HIV infection for women in subSaharan Africa is conflicting. A systematic review showed a significant bivariate association between early sexual debut and HIV in higher quality studies, while other studies found either that later risky sexual behavior instead contributed to infection risk, or that increased infection was explained by biological factors, including genital trauma at sexual debut resulting from physically forced sex [37].

A number of limitations are associated with this study. Due to convenience sampling, women in our study may not be representative of women 18-34 years of age living in Kisumu County; generalizability is an issue. We focused on women already using DMPA and OCPs; thus, it is unknown if women using other contraceptive methods or those without prior contraceptive use experience may have characteristics that differ from our sample. Our findings can only provide insights regarding women's notional acceptance of a contraceptive IVR; subsequent analyses are required to examine actual use and adherence. While women neither received eligibility criteria in advance of pre-screening nor were given specific reasons for ineligibility, there is the possibility that their overwhelming willingness to switch to the ring at pre-screening was influenced by social desirability. In addition, some women may have recognized or learned from others that willingness to use the ring was an eligibility requirement and that by providing a "yes" response this would help increase the likelihood that they would get into the study. Our recruitment method, while consistent with strategies for informing the community about happenings, may have prompted women to present for prescreening to avoid drawing attention to them by responding differently than their peers. It may have also minimized peer speculations regarding a woman's pregnancy or HIV status.

\section{Conclusion}

High notional acceptance suggests feasibility for contraceptive IVR use. Factors associated with actual ring will use need to be assessed.To address the high HIV and STI prevalence among young women in this setting, the co-formulation of hormonal contraception with antimicrobials may have enhanced uptake compared to rings for either indication alone.

\section{List of abbreviations \\ ARV: Antiretroviral \\ $\mathrm{Cl}$ : Confidence interval \\ DMPA: Depot medroxy-progesterone acetate}

ESR: Enrollment to screening ratio

FEM-PrEP: Pre-exposure Prophylaxis Trial for HIV Prevention among African Women

HIV: Human Immunodeficiency Virus

HSV-2: Herpes simplex virus type 2

IVRs: Intravaginal rings

MPTs: Multipurpose prevention technologies

OCP: Oral contraceptive pill

PR: Prevalence Ratio

STIs: Sexually transmitted infections

TDF/FTC: Tenofovir/emtricitabine

US: United States

VOICE: Vaginal and Oral Interventions to Control the Epidemic Trial

\section{Competing interests}

The authors declare that they have no competing interests.

\section{Authors' contributions}

\begin{tabular}{|l|c|c|c|c|c|c|c|c|c|c|}
\hline $\begin{array}{l}\text { Authors' } \\
\text { contributions }\end{array}$ & EM & DG & RG & MF & FO & MD & CZ & TS & BN & EM \\
\hline $\begin{array}{l}\text { Research concept } \\
\text { and design }\end{array}$ & $\checkmark$ & $\checkmark$ & -- & $\checkmark$ & $\checkmark$ & $\checkmark$ & $\checkmark$ & $\checkmark$ & $\checkmark$ & $\checkmark$ \\
\hline $\begin{array}{l}\text { Collection and/or } \\
\text { assembly of data }\end{array}$ & -- & -- & -- & $\checkmark$ & -- & -- & -- & -- & $\checkmark$ & $\checkmark$ \\
\hline $\begin{array}{l}\text { Data analysis and } \\
\text { interpretation }\end{array}$ & $\checkmark$ & $\checkmark$ & $\checkmark$ & $\checkmark$ & -- & -- & -- & -- & -- & -- \\
\hline Writing the article & $\checkmark$ & $\checkmark$ & $\checkmark$ & $\checkmark$ & $\checkmark$ & $\checkmark$ & $\checkmark$ & $\checkmark$ & $\checkmark$ & $\checkmark$ \\
\hline $\begin{array}{l}\text { Critical revision of } \\
\text { the article }\end{array}$ & $\checkmark$ & $\checkmark$ & $\checkmark$ & $\checkmark$ & -- & -- & -- & -- & -- & -- \\
\hline $\begin{array}{l}\text { Final approval of } \\
\text { article }\end{array}$ & $\checkmark$ & $\checkmark$ & $\checkmark$ & $\checkmark$ & $\checkmark$ & $\checkmark$ & $\checkmark$ & $\checkmark$ & $\checkmark$ & $\checkmark$ \\
\hline Statistical analysis & $\checkmark$ & $\checkmark$ & $\checkmark$ & -- & -- & -- & -- & -- & -- & -- \\
\hline
\end{tabular}

Acknowledgement and funding

The findings and conclusions in this report are those of the authors and do not necessarily represent the views of the 
Centers for Disease Control and Prevention. This manuscript is published with the permission of the KEMRI Director and the KEMRI Publication Review Committee. With the exception of personal identifying information, Eleanor McLellan-Lemal and Roman Gvetadze have full access to all the data in the study and take responsibility for the integrity of the data and the accuracy of the data analysis. First and foremost, we would like to extend our appreciation to the women who took part in the study and the broader Kisumu community. We thank Kenneth Ondeng'e and members of the community engagement team for their efforts in recruiting and following study participants, as well as their contributions in keeping Kisumu elected and provincial leaders, the general community, local community based-organizations, and local special interests groups informed about the conduct, implementation, and close out of the study. To Lawrence Opado and Richard Ndivo, we are indebted to their questionnaire programming skills and development of both a robust data management system and process for verifying and transferring complex data in real time. To Victor Mudhune, we are grateful for his handling of pharmaceutical issues and overseeing import, approval of NuvaRing for study purposes, and dispensation of rings to study participants. To the numerous KEMRI personnel (clinicians, counselors, data collectors, receptionist, laboratory technicians, etc.) specially assigned to work on the study or were willing to lend their support and expertise when waiting rooms filled or scheduling challenges arose. Lastly, we thank Drs. Clyde Hart and Charles LeBaron for their technical guidance and support. A special thanks to Lisa A. Mills for her contributions to the conceptualization of the study.

Publication history

Editor: Erich Cosmi, University of Padua, Italy.

EIC: Robert R. Freedman, Wayne State University, USA.

Received: 24-May-2016 Final Revised: 14-Jun-2016

Accepted: 30-Jun-2016 Published: 06-Jul-2016

\section{References}

1. Brady $M$ and Manning J. Lessons from reproductive health to inform multipurpose prevention technologies: don't reinvent the wheel. Antiviral Res. 2013; 100 Suppl:S25-31. | Article | PubMed

2. Fernandez-Romero JA, Deal C, Herold BC, Schiller J, Patton D, Zydowsky T, Romano J, Petro CD and Narasimhan M. Multipurpose prevention technologies: the future of HIV and STI protection. Trends Microbiol. 2015; 23:429-36. | Article | PubMed

3. Alexander NJ, Baker E, Kaptein M, Karck U, Miller L and Zampaglione E. Why consider vaginal drug administration? Fertil Steril. 2004; 82:1-12. Article | PubMed

4. Brady $\mathrm{M}$ and Tolley $\mathrm{E}$. Aligning product development and user perspectives: social-behavioural dimensions of multipurpose prevention technologies. BJOG. 2014; 121 Suppl 5:70-8. | Article | PubMed

5. Romano JW, Van Damme $L$ and Hillier $S$. The future of multipurpose prevention technology product strategies: understanding the market in parallel with product development. BJOG. 2014; 121 Suppl 5:15-8. | Article | PubMed

6. Woodsong C, MacQueen K, Amico KR, Friedland B, Gafos M, Mansoor L, Tolley $E$ and McCormack $S$. Microbicide clinical trial adherence: insights for introduction. J Int AIDS Soc. 2013; 16:18505. | Article | PubMed Abstract | PubMed FullText

7. Dieben TO, Roumen FJ and Apter D. Efficacy, cycle control, and user acceptability of a novel combined contraceptive vaginal ring. Obstet Gynecol. 2002; 100:585-93. | Article | PubMed

8. Montgomery ET, van der Straten A, Cheng H, Wegner L, Masenga G, von Mollendorf C, Bekker L, Ganesh S, Young K, Romano J, Nel A and Woodsong C. Vaginal ring adherence in sub-Saharan Africa: expulsion, removal, and perfect use. AIDS Behav. 2012; 16:1787-98. | Article | PubMed

9. Roumen FJ and Mishell DR, Jr. The contraceptive vaginal ring, NuvaRing((R)), a decade after its introduction. Eur J Contracept Reprod Health Care. 2012; 17:415-27. | Article | PubMed

10. van der Straten A, Montgomery ET, Cheng $H$, Wegner L, Masenga G, von Mollendorf C, Bekker L, Ganesh S, Young K, Romano J, Nel A and Woodsong $C$. High acceptability of a vaginal ring intended as a microbicide delivery method for HIV prevention in African women. AIDS Behav. 2012; 16:1775-86. | Article | PubMed

11. Woodsong $C$ and Holt JD. Acceptability and preferences for vaginal dosage forms intended for prevention of HIV or HIV and pregnancy. Adv Drug Deliv Rev. 2015; 92:146-54. | Article | PubMed

12. Brache $V$ and Faundes $A$. Contraceptive vaginal rings: a review. Contraception. 2010; 82:418-27. | Article | PubMed

13. Schurmans C, De Baetselier I, Kestelyn E, Jespers V, Delvaux T, Agaba SK, van Loen H, Menten J, van de Wijgert J and Crucitti T. The ring plus project: safety and acceptability of vaginal rings that protect women from unintended pregnancy. BMC Public Health. 2015; 15:348. | Article | PubMed Abstract | PubMed FullText

14. Ahrendt HJ, Nisand I, Bastianelli C, Gomez MA, Gemzell-Danielsson K, Urdl W, Karskov B, Oeyen L, Bitzer J, Page G and Milsom I. Efficacy, acceptability and tolerability of the combined contraceptive ring, NuvaRing, compared with an oral contraceptive containing $\mathbf{3 0}$ microg of ethinyl estradiol and $\mathbf{3} \mathbf{~ m g}$ of drospirenone. Contraception. 2006; 74:451-7. | Article | PubMed

15. Rosenberg ZF and Devlin B. Future strategies in microbicide development. Best Pract Res Clin Obstet Gynaecol. 2012; 26:503-13. | Article | PubMed

16. NASCOP. National Guidelines for HIV Testing and Counselling in Kenya. National AIDS and STI Control Programme, Ministry of Public Health and Sanitationl, Kenya: Nairobi, Kenya. 2008.

17. Fiddes $P$, Scott A, Fletcher J and Glasier A. Attitudes towards pelvic examination and chaperones: a questionnaire survey of patients and providers. Contraception. 2003; 67:313-7. | Article | PubMed

18. Van Lith LM, Yahner M and Bakamjian L. Women's growing desire to limit births in sub-Saharan Africa: meeting the challenge. Glob Health Sci Pract. 2013; 1:97-107. | Article | PubMed Abstract | PubMed FullText

19. Hindin MJ and Fatusi AO. Adolescent sexual and reproductive health in developing countries: an overview of trends and interventions. Int Perspect Sex Reprod Health. 2009; 35:58-62. | Article | PubMed

20. Akelo V, McLellan-Lemal E, Toledo L, Girde S, Borkowf CB, Ward L, Ondenge K, Ndivo R, Lecher SL, Mills LA and Thomas TK. Correction: Determinants and Experiences of Repeat Pregnancy among HIVPositive Kenyan Women--A Mixed-Methods Analysis. PLoS One. 2015; 10:e0134536. | Article | PubMed Abstract | PubMed FullText

21. Ikamari $L$ and $R$. Towett. Sexual initiation and contraceptive use among female adolescents in Kenya. African Journal of Health Sciences. 2008; 14:1-13. | Pdf

22. Brown $A$ and W.H. Organization. Sexual relations among young people in developing countries: evidence from WHO case studies. Geneva: World Health Organization, Department of Reproductive Health and Research. 2001.

23. Exavery A, Kante AM, Jackson E, Noronha J, Sikustahili G, Tani K, Mushi HP, Baynes C, Ramsey K, Hingora A and Phillips JF. Role of condom negotiation on condom use among women of reproductive age in three districts in Tanzania. BMC Public Health. 2012; 12:1097. | Article | PubMed Abstract | PubMed FullText

24. Ochako R, Mbondo M, Aloo S, Kaimenyi S, Thompson R, Temmerman M and Kays $\mathrm{M}$. Barriers to modern contraceptive methods uptake among young women in Kenya: a qualitative study. BMC Public Health. 2015; 15:118. | Article | PubMed Abstract | PubMed FullText

25. Dynes $M$, Stephenson $R$, Rubardt $M$ and Bartel $D$. The influence of perceptions of community norms on current contraceptive use among men and women in Ethiopia and Kenya. Health Place. 2012; 18:766-73. | Article | PubMed

26. Roddy RE, Zekeng L, Ryan KA, Tamoufe U, Weir SS and Wong EL. 
McLellan-Lemal et al. Research Journal of Women's Health 2016, http://www.hoajonline.com/journals/pdf/2054-9865-3-1.pdf

A controlled trial of nonoxynol 9 film to reduce male-to-female transmission of sexually transmitted diseases. N Engl J Med. 1998; 339:504-10. | Article | PubMed

27. Secura GM, Allsworth JE, Madden T, Mullersman JL and Peipert JF. The Contraceptive CHOICE Project: reducing barriers to long-acting reversible contraception. Am J Obstet Gynecol. 2010; 203:115 e1-7. | Article | PubMed Abstract | PubMed FullText

28. Abdel-Aleem $\mathrm{H}$ et al. Treatment of vaginal bleeding irregularities induced by progestin only contraceptives. The Cochrane Library. 2015.

29. Mantell JE, Myer L, Carballo-Dieguez A, Stein Z, Ramjee G, Morar NS and Harrison PF. Microbicide acceptability research: current approaches and future directions. Soc Sci Med. 2005; 60:319-30. | Article | PubMed

30. GoK. Kenya HIV prevention revolution road map: Countdown to 2030. N. Kilonzo, Editor. Ministry of Health National AIDS Control Council: Nairobi. 2014; 58.

31. van de Wijgert JH, Morrison CS, Brown J, Kwok C, Van Der Pol B, Chipato T, Byamugisha JK, Padian N and Salata RA. Disentangling contributions of reproductive tract infections to HIV acquisition in African Women. Sex Transm Dis. 2009; 36:357-64. | Article | PubMed

32. Esber A, Vicetti Miguel RD, Cherpes TL, Klebanoff MA, Gallo MF and Turner AN. Risk of Bacterial Vaginosis Among Women With Herpes Simplex Virus Type 2 Infection: A Systematic Review and Meta-analysis. $J$ Infect Dis. 2015; 212:8-17. | Article | PubMed

33. Gumbe A, McLellan-Lemal E, Gust DA, Pals SL, Gray KM, Ndivo R, Chen RT, Mills LA and Thomas TK. Correlates of prevalent HIV infection among adults and adolescents in the Kisumu incidence cohort study, Kisumu, Kenya. Int J STD AIDS. 2015; 26:929-40. | Article | PubMed Abstract | PubMed FullText

34. Amornkul PN, Vandenhoudt $H$, Nasokho P, Odhiambo F, Mwaengo D, Hightower A, Buve A, Misore A, Vulule J, Vitek C, Glynn J, Greenberg A, Slutsker $\mathrm{L}$ and De Cock KM. HIV prevalence and associated risk factors among individuals aged 13-34 years in Rural Western Kenya. PLoS One. 2009; 4:e6470. | Article | PubMed Abstract | PubMed FullText

35. Vodstrcil LA, Hocking JS, Law M, Walker S, Tabrizi SN, Fairley CK and Bradshaw CS. Hormonal contraception is associated with a reduced risk of bacterial vaginosis: a systematic review and meta-analysis. PLOS One. 2013; 8:e73055. | Article | PubMed Abstract | PubMed FullText

36. KNBS. Nyanza Province multiple indicator cluster survey 2011, final report. Nairobi, Kenya. 2013.

37. Stockl $\mathrm{H}$, Kalra N, Jacobi J and Watts $\mathrm{C}$. Is early sexual debut a risk factor for HIV infection among women in sub-Saharan Africa? A systematic review. Am J Reprod Immunol. 2013; 69 Suppl 1:27-40. | Article | PubMed

\section{Citation:}

McLellan-Lemal E, Gust DA, Gvetadze R, Furtado M, Otieno FO, Desai M, Zeh C, Samandari T, Nyagol B and Makang EM. Characteristics of women screened for a contraceptive intravaginal ring study in Kisumu, Kenya, 2014. Res J Womens Health. 2016; 3:1.

http://dx.doi.org/10.7243/2054-9865-3-1 